\title{
Simvastatin Attenuates Contrast-Induced Nephropathy through Modulation of Oxidative Stress, Proinflammatory Myeloperoxidase, and Nitric Oxide
}

\author{
Ketab E. Al-Otaibi, ${ }^{1}$ Abdulrahman M. Al Elaiwi, ${ }^{1}$ \\ Mohammad Tariq, ${ }^{2}$ and Abdulrahman K. Al-Asmari ${ }^{2}$ \\ ${ }^{1}$ Department of Urology, Prince Sultan Medical Military City, Riyadh, Saudi Arabia \\ ${ }^{2}$ Research Center, Prince Sultan Medical Military City, P.O. Box 7897 (S-775), Riyadh 11159, Saudi Arabia \\ Correspondence should be addressed to Abdulrahman K. Al-Asmari, abdulrahman.alasmari@gmail.com
}

Received 20 June 2012; Accepted 3 September 2012

Academic Editor: Sumitra Miriyala

Copyright $\odot 2012$ Ketab E. Al-Otaibi et al. This is an open access article distributed under the Creative Commons Attribution License, which permits unrestricted use, distribution, and reproduction in any medium, provided the original work is properly cited.

Contrast media- (CM-) induced nephropathy is a serious complication of radiodiagnostic procedures. Available data suggests that the development of prophylaxis strategies is limited by poor understanding of pathophysiology of CM-induced nephropathy. Present study was designed to determine the role of oxidative stress, myeloperoxidase, and nitric oxide in the pathogenesis of iohexol model of nephropathy and its modification with simvastatin (SSTN). Adult Sprague Dawley rats were divided into seven groups. After $24 \mathrm{~h}$ of water deprivation, all the rats except in control and SSTN-only groups were injected $(10 \mathrm{ml} / \mathrm{kg}) \mathrm{with} 25 \%$ glycerol. After $30 \mathrm{~min}$, SSTN $(15,30$, and $60 \mathrm{mg} / \mathrm{kg})$ was administered orally, daily for 4 days. Twenty-four hours after the glycerol injection, iohexol was infused $(8 \mathrm{ml} / \mathrm{kg})$ through femoral vein over a period of $2 \mathrm{~min}$. All the animals were sacrificed on day 5 and blood and kidneys were collected for biochemical and histological studies. The results showed that SSTN dose dependently attenuated CM-induced rise of creatinine, urea, and structural abnormalities suggesting its nephroprotective effect. A significant increase in oxidative stress (increased lipid hydroperoxides and reduced glutathione levels) and myeloperoxidase (MPO) and decreased nitric oxide in CM group were reversed by SSTN. These findings support the use of SSTN to combat CM-induced nephrotoxicity.

\section{Introduction}

Contrast media (CM) are frequently used for various diagnostic procedures including X-rays, computed tomography and magnetic resonance imaging. CM-induced nephropathy is a leading cause of acute kidney injury and is associated with significant mortality and morbidity $[1,2]$. The mechanism of CM-induced renal impairment at cellular level is not fully understood; recent reports suggested both direct and indirect effects on renal tubules including biochemical and hemodynamic disturbance due to CM-induced prerenal dehydration, hypotension, and medullary ischemia [3, 4]. Jost et al. [5] also reported that contrast media cause impairment of renal perfusion leading to hypoxic conditions resulting in acute tubular necrosis. It has been reported that patients with diabetes and preexisting renal insufficiency may be at a higher risk of CM-induced nephrotoxicity compared to patients with normal renal function [6].

Several experimental and clinical studies suggested beneficial effects of statins against CM-induced nephrotoxicity $[7,8]$; however, this claim has been refuted by some of the recent clinical trials $[9,10]$. Meta-analysis of available data about the renoprotective effect of statins against CMinduced nephropathy is still conflicting and inconclusive. It has been suggested that the development of highly effective prophylaxis strategies is limited by poor understanding of the pathophysiology of CM-induced renal toxicity. In this paper, attempt has been made to study the role of oxidative 
stress, proinflammatory enzyme myeloperoxidase (MPO), and vasoregulatory nitric oxide in the pathogenesis of CMinduced nephropathy and its modification with simvastatin (SSTN). Iohexol (CM) plus glycerol was administered in rats as a model for CM-induced nephrotoxicity according to the method described by Duan et al. [11]. Pretreatment with glycerol was used to simulate preexisting renal insufficiency in clinical setting; this protocol provides for an optimal nephropathy on exposure of rats to contrast media.

\section{Material and Methods}

2.1. Radio Contrast Medium and Drugs. Contrast medium, iohexol, was obtained from Amersham Health, Cork, Ireland. Glycerol was purchased from Sigma Chemical Co., St. Louis, USA. Simvastatin was purchased from Calbiochem, CA, USA.

2.2. Animals and Treatment Groups. Adult Sprague Dawley male rats $(250-300 \mathrm{~g})$ raised in our animal breeding facility were used in this study. The experimental protocol of this study was approved by Hospital Research and Ethical Committee.The animals were randomly divided into 7 groups (G) of 7 animals each; G1 (Control), G2 (Glycerol only), G3 (Glycerol + CM), G4 (Glycerol + CM + SSTN $15 \mathrm{mg} / \mathrm{kg}$ ), G5 (Glycerol + CM + SSTN $30 \mathrm{mg} / \mathrm{kg}$ ), G6 (Glycerol + CM + SSTN $60 \mathrm{mg} / \mathrm{kg}$ ), and G7 (SSTN $60 \mathrm{mg} / \mathrm{kg})$.

2.3. Study Protocol. The protocol suggested by Duan et al. [11] was followed in this study. After $24 \mathrm{~h}$ water deprivation, all rats except the control group (G1) and SSTN only group (G7) were injected intramuscularly with $10 \mathrm{~mL} / \mathrm{kg}$ body weight of $25 \%$ glycerol; drinking water and food were then resumed ad libitum. After 30 minutes of glycerol injection, SSTN was administered orally to the animals in groups G4, G5, G6, and G7; the different doses of SSTN were prepared in a volume of $10 \mathrm{~mL} / \mathrm{kg}$ and administered by gavage using an intubation needle. SSTN treatment was continued daily for 4 days. Twenty-four hours after the glycerol injection, CM were infused through femoral vein in the dosage of $8 \mathrm{~mL} / \mathrm{kg}$ of body weight over a period of $2 \mathrm{~min}$ to the animals in groups G3, G4, G5, and G6. All the animals were weighed and sacrificed on day 5 . Animals were killed under deep ethyl ether anaesthesia. Blood was collected through heart puncture and serum separated for renal function test. The kidneys were excised and weighed immediately; one kidney was stored at $-70^{\circ} \mathrm{C}$ for biochemical analysis and the other kidney was preserved in $10 \%$ formalin for histopathological studies as shown in Table 1 .

2.4. Renal Function Test. Blood urea nitrogen (BUN), serum creatinine (SCr), and serum calcium levels were measured spectrophotometrically (APEL PD-303S Japan) using the commercial kits from United Diagnostics Industry, Riyadh, Saudi Arabia. These values are expressed as mg/dL (Figure 1).
2.5. Determination of Lipid Peroxidation. The level of lipid peroxidation in the kidney tissue was assessed as thiobarbituric acid reactive substances (TBARS) according to the method of Ohkawa et al. [12]. The kidney tissue $(0.5 \mathrm{~g})$ was homogenized in $1.15 \%$ cold $\mathrm{KCl}$ using an Ultraturrax homogenizer (Janke and Kunkel, Staufen, Germany). The homogenate was centrifuged at $3000 \mathrm{~g}$ for $5 \mathrm{~min}$ and an aliquot of supernatant was mixed with $2 \mathrm{~mL}$ of reaction mixture (15\% trichloroacetic acid and $0.375 \%$ thiobarbituric acid in $0.25 \mathrm{~N} \mathrm{HC1}$ ) and heated for 5 minutes in a boiling water bath. The tubes were cooled at room temperature and centrifuged at $1000 \mathrm{~g}$ for 10 minutes. The absorbance of supernatant was read at $535 \mathrm{~nm}$ on a UV visible spectrophotometer (UV-160A, Shimadzu, Japan), against a blank that contained all reagents except homogenate. Tissue lipid peroxide levels were calculated as nanomoles of MDA using tetramethoxypropane as standard.

2.6. Determination of Glutathione. The reduced glutathione (GSH) in the kidney tissue was measured enzymatically according to the method of Mangino et al. [13]. The kidney tissue was homogenized with $1 \mathrm{~mL}$ of $0.1 \mathrm{M}$ perchloric acid plus $0.005 \%$ EDTA in a ratio of $1: 20$ (wt/vol). The homogenate was centrifuged at $4000 \mathrm{rpm}$ for $10 \mathrm{~min}$ and the supernatant was used for GSH assay. The reaction mixture consisted of the following freshly prepared solutions: solu-

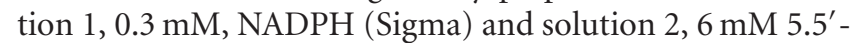
dithio-bis (2-nitrobenzoic acid) (Sigma), and solution 3, $50 \mathrm{U} / \mathrm{mL}$ glutathione reductase (Sigma). All three solutions were prepared with a stock buffer consisting of $125 \mathrm{mM}$ $\mathrm{NaH}_{2} \mathrm{PO}_{4}$ and $6.3 \mathrm{mM}$ EDTA at $\mathrm{pH}$ 7.5. For GSH assay, $800 \mu \mathrm{L}$ of solution $1,100 \mu \mathrm{L}$ of solution 2 , and $10 \mu \mathrm{L}$ of solution-3 were mixed in a quartz cuvette and placed in a UV-VIS spectrophotometer at $30^{\circ} \mathrm{C}$. The enzymatic reaction was started by the addition of $100 \mu \mathrm{L}$ of the sample (the supernatant) and the absorbance was monitored for $3 \mathrm{~min}$ at $412 \mathrm{~nm}$ on a UV-visible Spectrophotometer (UV-160A, Shimadzu, Japan). The slope of the change in absorbance was used to quantitate GSH by comparing the slope of the samples with a standard curve prepared with pure GSH (Sigma).

2.7. Determination of Myeloperoxidase. The activity of inflammatory marker myeloperoxidase (MPO) in the tissue was measured according to the method of Barone et al. [14]. Preweighed tissue was homogenized (1:20 wt/vol) in $5 \mathrm{mM}$ potassium phosphate buffer ( $\mathrm{pH}$ 6.0) using an Ultraturrax homogenizer. The homogenate was centrifuged at $17000 \mathrm{~g}$ for $15 \mathrm{~min}$ at $4^{\circ} \mathrm{C}$. The supernatant was discarded, and the pellet was extracted with $0.5 \%$ hexadecyltrimethylammonium bromide (Sigma) in $50 \mathrm{mM}$ potassium phosphate buffer ( $\mathrm{pH}$ 6.0). Three freeze-thaw cycles were performed, with sonication $\left(10 \mathrm{~s}, 25^{\circ} \mathrm{C}\right)$ between cycles. After the last sonication, the samples were incubated at $4^{\circ} \mathrm{C}$ for 2 minutes and centrifuged at $12500 \mathrm{~g}\left(15 \mathrm{~min}, 4^{\circ} \mathrm{C}\right)$. MPO in the supernatant was assayed by mixing $0.1 \mathrm{~mL}$ of supernatant with $2.9 \mathrm{~mL}$ of $50 \mathrm{mM}$ potassium phosphate buffer $(\mathrm{pH}$ 6.0) containing $0.167 \mathrm{mg} / \mathrm{mL}$ o-dianisidine dihydrochloride 
TABLe 1: Drug treatment protocol.

\begin{tabular}{|c|c|c|c|c|c|c|}
\hline Groups & Day 0 & Day 1 & Day 2 & Day 3 & Day 4 & Day 5 \\
\hline Control (G-1) & WD & Saline & Saline & - & - & Sacrificed \\
\hline Glycerol (G-2) & WD & Gly + & Saline & - & - & Sacrificed \\
\hline CM + Gly (G-3) & WD & Gly & $\mathrm{CM}$ & - & - & Sacrificed \\
\hline $\mathrm{CM}+$ Gly + SSTN $15(\mathrm{G}-4)$ & WD & Gly + SSTN & $\mathrm{CM}+\mathrm{SSTN}$ & SSTN & SSTN & Sacrificed \\
\hline $\mathrm{CM}+\mathrm{Gly}+\mathrm{SSTN} 30(\mathrm{G}-5)$ & WD & Gly + SSTN & $\mathrm{CM}+\mathrm{SSTN}$ & SSTN & SSTN & Sacrificed \\
\hline $\mathrm{CM}+\mathrm{Gly}+\mathrm{SSTN} 60(\mathrm{G}-6)$ & WD & Gly + SSTN & $\mathrm{CM}+\mathrm{SSTN}$ & SSTN & SSTN & Sacrificed \\
\hline SSTN 60 (G-7) & WD & SSTN & SSTN & SSTN & SSTN & Sacrificed \\
\hline
\end{tabular}

Abbreviations: WD (Water deprived), Gly (Glycerol), CM (Contrast Media), and SSTN (Simvastatin).

Blood urea nitrogen
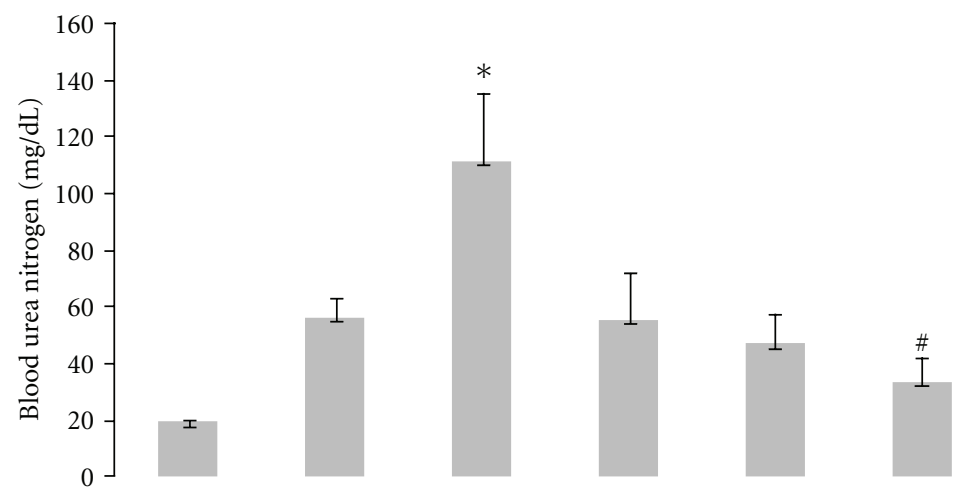

Serum creatinine
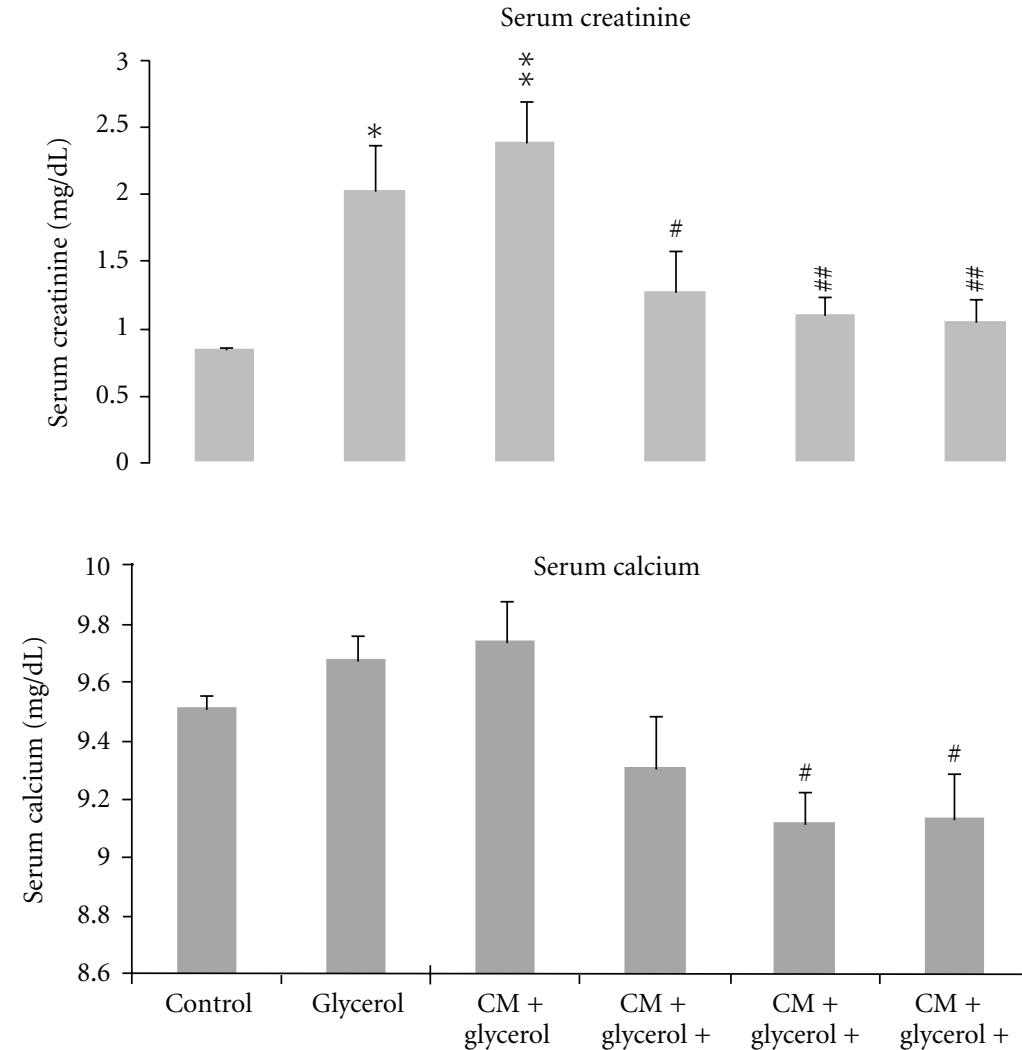

Serum calcium
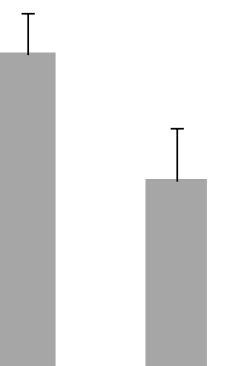

$\mathrm{CM}+$ glycerol

$\mathrm{CM}+$ glycerol + SSTN15 glycerol SSTN30

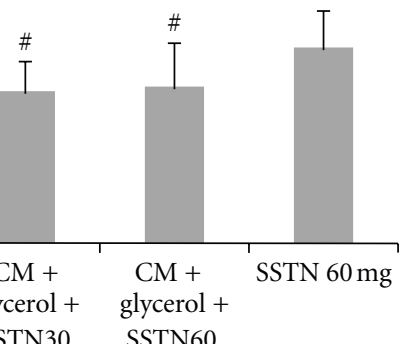

FIGURE 1: Effect of Simvastatin treatment on blood urea nitrogen, serum creatinine, and calcium in a rat model of contrast media-induced nephropathy. Values are \pm SEM. ${ }^{*} P<0.05,{ }^{* *} P<0.01$ as compared with control group, and ${ }^{\#} P<0.05$, ${ }^{\#} P<0.01$ versus glycerol $+\mathrm{CM}$ using Tukey's test. CM: contrast media; SSTN: simvastatin, 15, 30, and $60 \mathrm{mg} / \mathrm{kg}$. 
(Sigma) and $0.0005 \%$ hydrogen peroxide (ICN Pharmaceuticals, Irvine, CA). The change in absorbance at $460 \mathrm{~nm}$ was measured for $3 \mathrm{~min}$ by using a UV-visible spectrophotometer (UV-160A, Shimadzu, Japan).

2.8. Determination of Nitric Oxide. The levels of nitric oxide (NO) in kidney tissues were determined indirectly using the Griess reaction for simultaneous evaluation of nitrite and nitrate concentrations as described by Moshage et al. [15]. Kidney tissue was homogenized in $0.1 \mathrm{M}$ sodium phosphate buffer $\mathrm{pH} 7.4$ in $1: 10(\mathrm{w} / \mathrm{v})$ and centrifuged at $8000 \mathrm{~g}$ for 10 minutes. Deproteinization of the sample was carried out by the mixing of $40 \mu \mathrm{L}$ of $30 \mathrm{~g} / 100 \mathrm{~mL}$ zinc sulphate, in $800 \mu \mathrm{L}$ of sample using vortex and the solution was allowed to stand for $10 \mathrm{~min}$. The tubes were centrifuged at $4000 \mathrm{~g}$ for $10 \mathrm{~min}$. For nitrite assay, $500 \mu \mathrm{L}$ of the clear deproteinized supernatant were transferred into the tubes containing $400 \mu \mathrm{L}$ distilled water, $500 \mu \mathrm{L}$ glycine buffer, and $1 \mathrm{~mL}$ of Griess reagent $(1 \mathrm{~g} / \mathrm{L}$ sulfanilamide, $25 \mathrm{~g} / \mathrm{L}$ phosphoric acid, and $0.1 \mathrm{~g} / \mathrm{L} \mathrm{N}-1$ naphthylethylenediamine). After 15-20 min the absorbance was measured at $545 \mathrm{~nm}$ against a blank containing all the reagents except the homogenate sample. For nitrate estimation the same steps were followed but before addition of Griess reagent 2.5-3 g cadmium granules were added in every tube and the samples were kept for 2 hours at room temperature and the absorbance was read at $545 \mathrm{~nm}$ on a UV-visible Spectrophotometer (UV-160A, Shimadzu, Japan). Calculation of nitric oxide was based on nitrite and nitrate values.

2.9. Kidney Weight-to-Body Weight Ratio. At the time of sacrifice the body weights and the kidney weights of rats were recorded. The kidney weight body weight ratio was calculated by simple arithmetic calculation of the kidney weight divided by body weight and then converted to percent.

2.10. Histopathological Examination. After recording the kidney weight and morphological examination, the kidney was fixed immediately in $10 \%$ formalin, embedded in paraffin, sectioned at $3 \mu \mathrm{m}$ thickness and the sections were stained with hematoxylin and eosin. The extents of tubular injury, dilatation, vacuolation, and necrosis were evaluated semiquantitatively with a slight modification of the method of Raij et al. [16]. Briefly, the extent of tissue damage was graded from $0-4$ according to the severity of tubular necrosis, tubular vacuolation, and tubular dilatation. The scoring system was as follows: $0=$ no change in the tubules, $1 \leq$ $25 \%$ of tubular injury (mild), $2=25 \%$ to $50 \%$ of tubular involvement (moderate), $3=50 \%$ to $75 \%$ of tubules showing characteristic change (severe), and $4 \geq 75 \%$ of tubular damage (very severe). Fifty fields were counted from each slide. All the assessments were done in a blinded fashion.

2.11. Statistics. The data were evaluated by one-way analysis of variance (ANOVA) followed by Tukey's test using SPSS (Version 15) statistical package. $P$ values $<0.05$ were considered as statistically significant.

\section{Results}

Treatment of rats with SSTN alone did not produce any significant change in serum BUN, SCr, and calcium levels (Figure 1). Administration of Glycerol alone increased the serum BUN levels which were significantly potentiated by CM (ANOVA $F=3.820, P<0.01$ ). Treatment with SSTN dose dependently attenuated CM-induced BUN levels (Figure 1). Serum creatinine was significantly increased by glycerol as well as glycerol + CM (ANOVA $F=7.259$, $P<0.001)$. Treatment with SSTN significantly and dose dependently reduced the SCr levels induced by glycerol + $\mathrm{CM}$ and also in SSTN-treated control animals (Figure 1). Treatment of rats with glycerol alone and glycerol plus CM produced a nonsignificant increase in serum calcium. Both the medium $(30 \mathrm{mg} / \mathrm{kg})$ and high doses $(60 \mathrm{mg} / \mathrm{kg})$ of SSTN were equipotent in reducing serum calcium levels in glycerol + CM treated rats (ANOVA $F=3.702, P<0.01$ ) (Figure 1 ).

Administration of Glycerol with or without CM significantly increased TBARS levels in kidneys of rats which were significantly attenuated by medium and high doses of SSTN (ANOVA $F=15.239, P<0.001$ ) (Figure 2). The level of kidney GSH significantly decreased in rats exposed to glycerol or glycerol plus CM. Treatment with medium and high doses of statin significantly and dose dependently reversed the depletion of GSH caused by glycerol and CM (ANOVA $F=16.223, P<0.001$ ). Although glycerol alone produced a nonsignificant increase of the activity of MPO in kidneys, combined treatment with glycerol plus CM significantly increased MPO activity (Figure 2) which was significantly reduced by high dose of SSTN (ANOVA $F=$ 6.380, $P<0.001)$. A significant reduction in kidney NO level was observed in rats exposed to glycerol alone or in combination with CM. Treatment with medium and high doses of SSTN significantly reversed the effects of Glycerol and CM on kidney NO levels (ANOVA $F=6.008, P<0.001$ ).

Administration of glycerol alone or in combination with CM significantly increased kidney weight to body weight ratio (ANOVA $F=10.228, P<0.001$ ). Concomitant treatment with SSTN reduced glycerol and CM-induced increase in kidney/body weight ratio; however, the reduction was not significant (Table 2).

3.1. Histological Findings. The light microscopic findings in the kidneys of different treatment groups are given in Table 2. The groups injected with only glycerol produced tubular dilatation and mild tubular vacuolation and necrosis, whereas treatment with glycerol and CM produced significant tubular dilatation, tubular vacuolation, and tubular necrosis. Treatment of rats with SSTN dose dependently reversed CM-induced histological changes (Table 2, Figure 3).

\section{Discussions}

The findings of this study clearly showed a significant increase in the biomarkers of renal injury including BUN and $\mathrm{SCr}$ in the rats exposed to $\mathrm{CM}$ plus glycerol (Figure 1). The increase in SCr and BUN following exposure of rats 

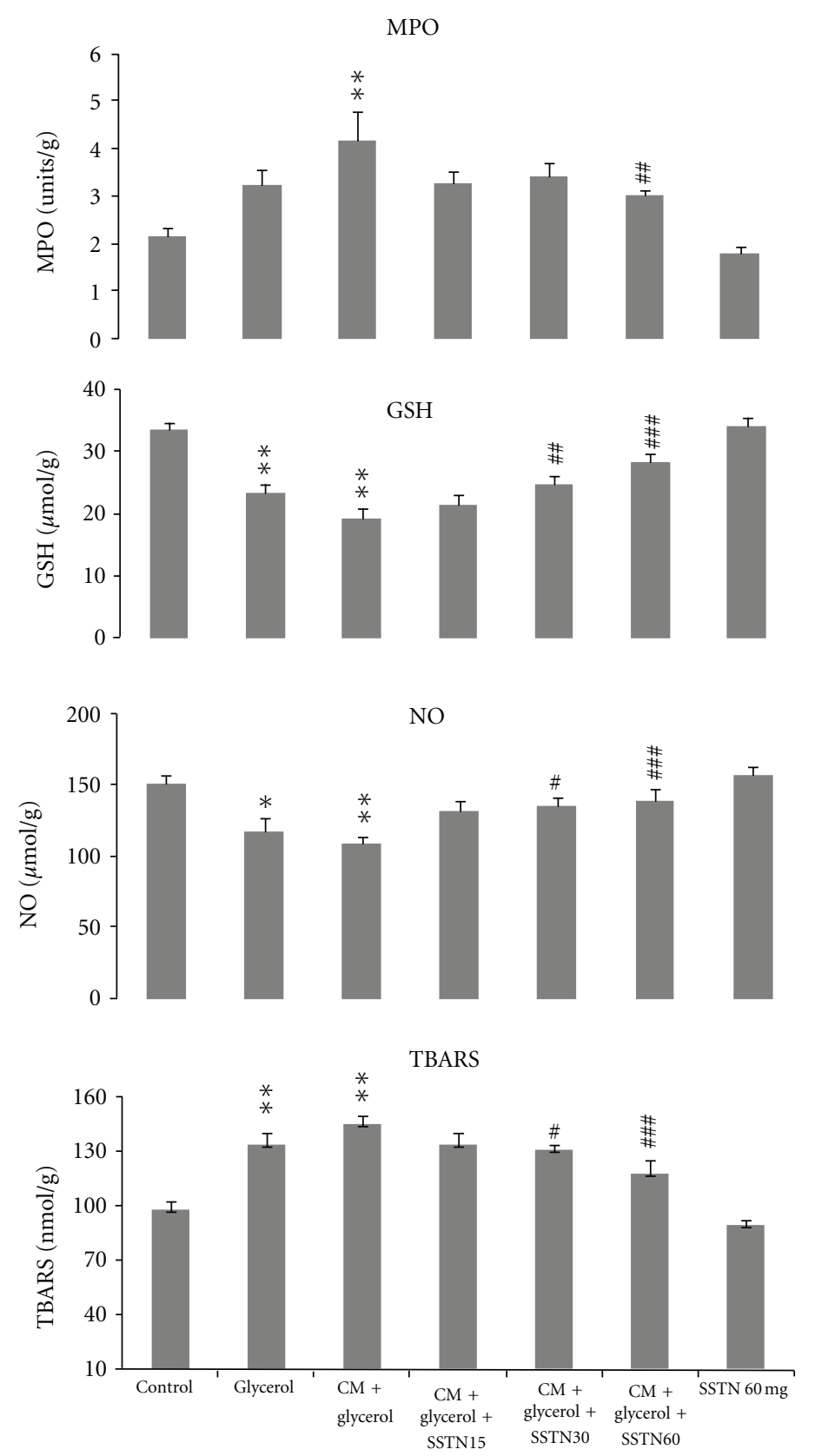

FIGURE 2: Effect of simvastatin on TBARS, NO, GSH, and MPO in a rat model of contrast media-induced nephropathy. Values are mean \pm SEM. ${ }^{*} P<0.05,{ }^{* *} P<0.01$ as compared to control group and ${ }^{\#} P<0.05,{ }^{\# \#} P<0.01$, and ${ }^{\# \# \#} P<0.001$ versus glycerol + CM group using Tukey's test. CM: contrast media; SSTN: simvastatin 10, 30, and $60 \mathrm{mg} / \mathrm{kg}$.

to $\mathrm{CM}$ and glycerol has been reported earlier $[17,18]$. Our histopathological studies also confirm significant tubular vacuolar transformation, interstitial edema, and tubular degeneration in this nephropathy model. Histologic alterations including proximal tubular vacuolar transformation, interstitial edema, and tubular degeneration following contrast administration have also been reported earlier [19]. Development of edema and enlargement of kidney by CM which was evident by significant increase in kidney weightto-body weight ratio also confirm significant renal toxicity (Figure 3). Pretreatment with SSTN significantly and dose dependently reversed the effects of $\mathrm{CM}$ on serum markers of renal injury (Figure 1). Treatment of animals with SSTN has been shown to exert a favorable effect on renal blood flow and glomerular filtration rate by earlier investigators [20], that is evident from a decrease in serum creatinine and blood urea nitrogen levels even in control rats treated with SSTN (Figure 1). There was a nonsignificant increase in the level of serum calcium following exposure to contrast media (Figure 1). On the other hand, treatment with SSTN prevented contrast induced rise in serum calcium. Calcium plays an important role in maintaining cell viability and 


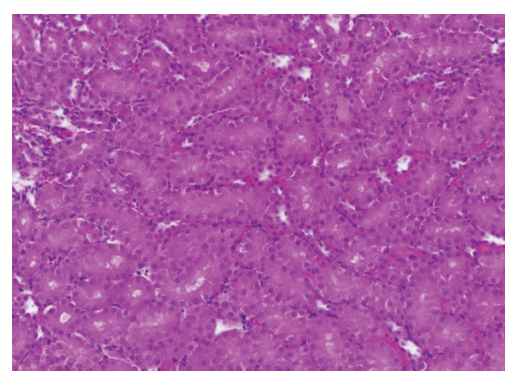

(A)

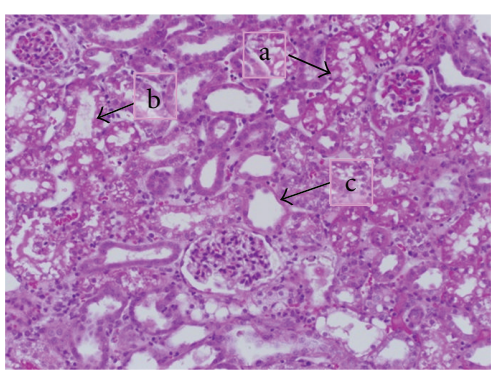

(B)

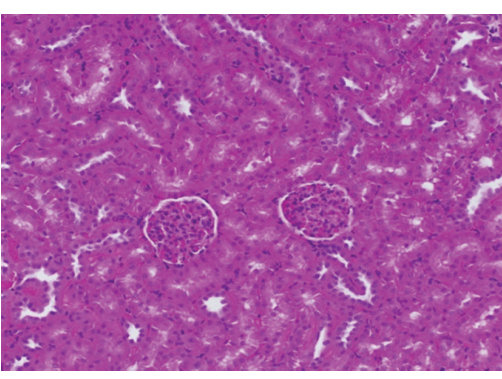

(C)

Figure 3: Light microscopy of renal tissue of rats stained with hematoxylin and eosin $(\times 200)$. (A) Normal histology of kidney tissue. (B) Marked tubular necrosis (a), dilatation (b), and vacuolation (c) in CM model of nephropathy. (C) SSTN-induced reversal of CM-induced nephropathy showing minimal tubular abnormalities.

TABLE 2: Effect of simvastatin on tubular injury scores in a rat model of CM nephropathy.

\begin{tabular}{lcccc}
\hline Groups & Tubular dilatation & Tubular vacuolation & Tubular necrosis & Kidney wt/Body wt ratio (\%) \\
\hline Control & 0 & 0 & 0 & $0.33 \pm 0.01$ \\
Glycerol & $2.7 \pm 0.28^{* * *}$ & $0.6 \pm 0.20^{*}$ & $0.70 \pm 0.18^{* *}$ & $0.58 \pm 0.04^{* * *}$ \\
CM + glycerol & $3.4 \pm 0.32^{* * *}$ & $2.9 \pm 0.29^{* * *}$ & $3.0 \pm 0.26^{* * *}$ & $0.61 \pm 0.04^{* * *}$ \\
CM + glycerol + SSTN 15 & $2.3 \pm 0.28^{\#}$ & $1.0 \pm 0.01^{\# \#}$ & $0.40 \pm 0.20^{\# \#}$ & $0.50 \pm 0.03^{\#}$ \\
CM + glycerol + SSTN 30 & $1.9 \pm 0.22^{\# \#}$ & $0.9 \pm 0.01^{\# \#}$ & $0 \pm 0^{\# \#}$ & $0.48 \pm 0.03^{\#}$ \\
CM + glycerol + SSTN 60 & $1.2 \pm 0.2^{\# \# \#}$ & $0.4 \pm 0.24^{\# \#}$ & 0 & $0.47 \pm 0.03^{\# \#}$ \\
SSTN 60 & 0 & 0 & 0 & $0.31 \pm 0.00$ \\
\hline
\end{tabular}

Values are mean \pm SEM. ${ }^{*} P<0.05,{ }^{* *} P<0.01$, and ${ }^{* * *} P<0.001$ versus control group; ${ }^{\#} P<0.05,{ }^{\# \#} P<0.01$, and ${ }^{\# \# \#} P<0.001$ versus glycerol $+\mathrm{CM}$ group using Tukey's test.

High scores indicate severe injury.

integrity and alteration of calcium homeostasis may lead to cellular injury through activation of phospholipases, impairment of ion permeability, and their subsequent effect on cytoskeletal structure and function. Calcium channel blocker amlodipine has been shown to attenuate CMinduced nephropathy [18].

The pathogenesis of CM-induced nephropathy is poorly understood; however, numerous pathways have been suggested to participate in the injury process. While some studies support a role for direct action of CM on tubular toxicity, other investigators suggested that the final pathway for contrast-induced renal injury might be due to medullary hypoxia conditioned by medullary ischemia (due to volume depletion and prostaglandin inhibition) affecting metabolically active and hyperfiltering nephrons. CM infusion induces renal vasoconstriction [3], a process that is thought to arise from an imbalance between endothelium-derived vasoconstrictive and vasodilatory factors $[21,22]$. Depending on the severity of CM induced vasoconstriction, ischemic tubular injury may result in cell detachment, apoptosis, or necrosis $[23,24]$. The ischemic insult is often accompanied by reperfusion injury involving excessive generation of potentially toxic-free radicals and inflammatory mediators [24].

We observed a significant increase in TBARS and decrease in GSH in kidneys of rats exposed to CM, indicating a state of oxidative stress (Figure 2). Treatment of rats with SSTN reversed CM-induced oxidative stress. A significant increase in lipid peroxides has been observed in kidneys of rats treated with CM [11]. Earlier studies also suggested that oxidative stress plays an important role in CM-induced nephrotoxicity [25-27]. The antioxidant, $\mathrm{N}$-acetylcysteine (NAC), has been used clinically for prevention of iothalamate meglumine contrast-induced acute renal failure [28-32]. The protective effect of SSTN against CM can be attributed to its antioxidant property.

There was a significant increase in the proinflammatory enzyme MPO levels in the kidneys of CM-treated rats; this rise in MPO levels was attenuated by SSTN (Figure 2). Increased MPO concentration in the kidney is an indicator of neutrophil infiltration and it is directly proportional to the tissue inflammation caused by CM. MPO activation has been shown to cause glomerular morphologic changes, endothelial and mesangial cell injury, and activation of platelets as well as glomerulonephritis [33]. Higher activity of MPO has been reported in primed macrophages under inflammatory conditions in both patients with chronic kidney disease and experimental studies [34]. The protective effect of SSTN against CM-induced increase in MPO levels can be attributed to its anti-inflammatory property [35]. It has been reported that SSTN may prevent loss of kidney function to a greater extent in individuals with evidence of increased inflammation than in those who show no inflammation [36].

Biochemical analysis of kidney showed a significant decrease in NO levels in our contrast media model of 
renal toxicity (Figure 2). A significant decrease in kidney NO following exposure of rats to iodixanol has also been reported by Sendeski et al. [37]. Decreased eNOS expression and increased plasma endothelin-1 have been implicated in nonionic CM-induced endothelial dysfunction and kidney injury [17]. Aggravation of CM-induced nephrotoxicity by hypercholesterolemia has been associated with the reduced production of $\mathrm{NO}$ [38]. Administration of L-arginine (NO donor) significantly attenuated hypercholesterolemiainduced aggravation of CM nephrotoxicity in rats [39]. Statins exert a positive influence on NO resulting in improved microcirculation, reduction in platelet aggregation, and enhancing cell proliferation [40-43]. Preservation of renal function by simvastatin in cyclosporine-induced ischemic/reperfusion injury has also been attributed to its vasodilator properties mediated by $\mathrm{NO}$ [44]. In conclusion SSTN offers significant nephroprotection against CMinduced nephropathies by inhibiting oxidative stress and inflammation and by improving renal microcirculation. Further clinical trials by using higher doses of statins alone or in combination with other nephroprotective agents should be tested as prophylactic agents to combat contrast-induced nephropathy.

\section{Conclusion}

In conclusion, the results of this study suggest a significant role of oxidative stress, proinflammatory myeloperoxidase and vasoregulatory nitric oxide in the pathogenesis of contrast-induced nephropathy. Treatment with high dose of SSTN prevents contrast-induced biochemical and structural changes in kidney. Therefore, further studies are warranted to test high doses of SSTN alone or in combination with other antioxidants and/or anti-inflammatory drugs to prevent CM-induced nephropathy.

\section{References}

[1] K. A. Sterling, T. Tehrani, and M. R. Rudnick, "Clinical significance and preventive strategies for contrast-induced nephropathy," Current Opinion in Nephrology and Hypertension, vol. 17, no. 6, pp. 616-623, 2008.

[2] N. Pannu, N. Wiebe, M. Tonelli, and Alberta Kidney Disease Network, "Prophylaxis strategies for contrast-induced nephropathy," The Journal of the American Medical Association, vol. 295, no. 23, pp. 2765-2779, 2006.

[3] L. S. Weisberg, P. B. Kurnik, and B. R. C. Kurnik, "Radiocontrast-induced nephropathy in humans: role of renal vasoconstriction," Kidney International, vol. 41, no. 5, pp. 1408-1415, 1992.

[4] I. Hizoh and C. Haller, "Radiocontrast-induced renal tubular cell apoptosis: hypertonic versus oxidative stress," Investigative Radiology, vol. 37, no. 8, pp. 428-434, 2002.

[5] G. Jost, H. Pietsch, J. Sommer et al., "Retention of iodine and expression of biomarkers for renal damage in the kidney after application of iodinated contrast media in rats," Investigative Radiology, vol. 44, no. 2, pp. 114-123, 2009.

[6] R. D. Moore, E. P. Steinberg, N. R. Powe et al., "Nephrotoxicity of high-osmolality versus low-osmolality contrast media: randomized clinical trial," Radiology, vol. 182, no. 3, pp. 649655, 1992.

[7] S. Acikel, H. Muderrisoglu, A. Yildirir et al., "Prevention of contrast-induced impairment of renal function by short-term or long-term statin therapy in patients undergoing elective coronary angiography," Blood Coagulation and Fibrinolysis, vol. 21, no. 8, pp. 750-757, 2010.

[8] S. Yoshida, H. Kamihata, S. Nakamura et al., "Prevention of contrast-induced nephropathy by chronic pravastatin treatment in patients with cardiovascular disease and renal insufficiency," Journal of Cardiology, vol. 54, no. 2, pp. 192198, 2009.

[9] L. Zhang, L. Zhang, Y. Lu et al., "Efficacy of statin pretreatment for the prevention of contrast-induced nephropathy: a metaanalysis of randomised controlled trials," International Journal of Clinical Practice, vol. 65, no. 5, pp. 624-630, 2011.

[10] M. A. Munoz, P. R. Maxwell, K. Green, D. W. Hughes, and R. L. Talbert, "Pravastatin versus simvastatin for prevention of contrast-induced nephropathy," Journal of Cardiovascular Pharmacology and Therapeutics, vol. 16, no. 3-4, pp. 376-379, 2011.

[11] S. B. Duan, F. Y. Liu, J. A. Luo et al., "Nephrotoxicity of high- and low-osmolar contrast media: the protective role of amlodipine in a rat model," Acta Radiologica, vol. 41, no. 5, pp. 503-507, 2000.

[12] H. Ohkawa, N. Ohishi, and K. Yagi, "Assay for lipid peroxides in animal tissues by thiobarbituric acid reaction," Analytical Biochemistry, vol. 95, no. 2, pp. 351-358, 1979.

[13] M. J. Mangino, M. K. Murphy, G. G. Grabau, and C. B. Anderson, "Protective effects of glycine during hypothermic renal ischemia-reperfusion injury," American Journal of Physiology, vol. 261, no. 5, part 2, pp. F841-F848, 1991.

[14] F. C. Barone, L. M. Hillegass, W. J. Price et al., "Polymorphonuclear leukocyte infiltration into cerebral focal ischemic tissue: myeloperoxidase activity assay and histologic verification," Journal of Neuroscience Research, vol. 29, no. 3, pp. 336$345,1991$.

[15] H. Moshage, B. Kok, J. R. Huizenga, and P. L. M. Jansen, "Nitrite and nitrate determinations in plasma: a critical evaluation," Clinical Chemistry, vol. 41, no. 6, part 1, pp. 892896, 1995.

[16] L. Raij, S. Azar, and W. Keane, "Mesangial immune injury, hypertension, and progressive glomerular damage in Dahl rats," Kidney International, vol. 26, no. 2, pp. 137-143, 1984.

[17] Y. Zhao, Z. Tao, Z. Xu et al., "Toxic effects of a high dose of non-ionic iodinated contrast media on renal glomerular and aortic endothelial cells in aged rats in vivo," Toxicology Letters, vol. 202, no. 3, pp. 253-260, 2011.

[18] O. Toprak, M. Cirit, M. Tanrisev et al., "Preventive effect of nebivolol on contrast-induced nephropathy in rats," Nephrology Dialysis Transplantation, vol. 23, no. 3, pp. 853-859, 2008.

[19] A. S. Berns, "Nephrotoxicity of contrast media," Kidney International, vol. 36, no. 4, pp. 730-740, 1989.

[20] S. R. Inman, T. W. Caprio, E. Drummond, M. Mueller, and K. Entenman, "Enhanced acetylcholine-induced dilation in afferent arterioles in simvastatin-fed rats," Vascular Pharmacology, vol. 44, no. 1, pp. 17-21, 2006.

[21] Y. Agmon, H. Peleg, Z. Greenfeld, S. Rosen, and M. Brezis, "Nitric oxide and prostanoids protect the renal outer medulla from radiocontrast toxicity in the rat," Journal of Clinical Investigation, vol. 94, no. 3, pp. 1069-1075, 1994.

[22] N. Klause, T. Arendt, M. Lins, and G. Gronow, "Hypoxic renal tissue damage by endothelin-mediated arterial vasoconstriction during radioangiography in man," Advances in 
Experimental Medicine and Biology, vol. 454, pp. 225-234, 1998.

[23] R. Beeri, Z. Symon, M. Brezis et al., "Rapid DNA fragmentation from hypoxia along the thick ascending limb of rat kidneys," Kidney International, vol. 47, no. 6, pp. 1806-1810, 1995.

[24] A. M. Sheridan and J. V. Bonventre, "Pathophysiology of ischemic acute renal failure," Contributions to Nephrology, vol. 132, pp. 7-21, 2001.

[25] A. Yesilyurt, I. Aydin Erden, I. Bilgiç, G. Erden, and A. Albayrak, "The protective effect of erdosteine on radiocontrast induced nephrotoxicity in rats," Environmental Toxicology, vol. 26, no. 4, pp. 395-402, 2011.

[26] Z. Parvez, M. A. Rahman, and R. Moncada, "Contrast mediainduced lipid peroxidation in the rat kidney," Investigative Radiology, vol. 24, no. 9, pp. 697-702, 1989.

[27] H. C. Lee, H. W. Yen, and S. H. Sheu, "Effects of different contrast media on glutathione peroxidase and superoxide dismutase activities in the heart and kidneys of normal and streptozotocin-induced diabetic rats," Journal of the Formosan Medical Association, vol. 105, no. 7, pp. 530-535, 2006.

[28] C. F. Pinto, M. Watanabe, and M. D. F. F. Vattimo, "Hydration and $\mathrm{N}$-acetylcysteine in acute renal failure caused by iodinated contrast medium: an experiment with rats," Journal of Nephrology, vol. 21, no. 5, pp. 783-788, 2008.

[29] A. Imaeda, T. Tanigawa, T. Aoki, Y. Kondo, N. Nakamura, and T. Yoshikawa, "Antioxidative effects of fluvastatin and its metabolites against oxidative DNA damage in mammalian cultured cells," Free Radical Research, vol. 35, no. 6, pp. 789801, 2001.

[30] M. Kassan, M. J. Montero, and M. A. Sevilla, "In vitro antioxidant activity of pravastatin provides vascular protection," European Journal of Pharmacology, vol. 630, no. 1-3, pp. 107$111,2010$.

[31] Y. An, H. Xin, W. Yan, and X. Zhou, "Amelioration of cisplatininduced nephrotoxicity by pravastatin in mice," Experimental and Toxicologic Pathology, vol. 63, no. 3, pp. 215-219, 2011.

[32] M. Fujieda, T. Morita, K. Naruse et al., "Effect of pravastatin on cisplatin-induced nephrotoxicity in rats," Human and Experimental Toxicology, vol. 30, no. 7, pp. 603-615, 2011.

[33] S. Sela, R. Shurtz-Swirski, M. Cohen-Mazor et al., "Primed peripheral polymorphonuclear leukocyte: a culprit underlying chronic low-grade inflammation and systemic oxidative stress in chronic kidney disease," Journal of the American Society of Nephrology, vol. 16, no. 8, pp. 2431-2438, 2005.

[34] M. R. Rodrigues, D. Rodriguez, M. Russo, and A. Campa, "Macrophage activation includes high intracellular myeloperoxidase activity," Biochemical and Biophysical Research Communications, vol. 292, no. 4, pp. 869-873, 2002.

[35] H. W. Yao, L. G. Mao, and J. P. Zhu, "Protective effects of pravastatin in murine lipopolysaccharide-induced acute lung injury," Clinical and Experimental Pharmacology and Physiology, vol. 33, no. 9, pp. 793-797, 2006.

[36] V. Krane and C. Wanner, "Statins, inflammation and kidney disease," Nature Reviews Nephrology, vol. 7, no. 7, pp. 385-397, 2011.

[37] M. Sendeski, A. Patzak, T. L. Pallone, C. Cao, A. E. Persson, and P. B. Persson, "Iodixanol, constriction of medullary descending vasa recta, and risk for contrast medium-induced nephropathy," Radiology, vol. 251, no. 3, pp. 697-704, 2009.

[38] D. W. Yang, R. H. Jia, D. P. Yang, G. H. Ding, and C. $\mathrm{X}$. Huang, "Dietary hypercholesterolemia aggravates contrast media-induced nephropathy," Chinese Medical Journal, vol. 117, no. 4, pp. 542-546, 2004.
[39] L. Andrade, S. B. Campos, and A. C. Seguro, "Hypercholesterolemia aggravates radiocontrast nephrotoxicity: protective role of L-arginine," Kidney International, vol. 53, no. 6, pp. 1736-1742, 1998.

[40] S. Wolfrum, K. S. Jensen, and J. K. Liao, "Endotheliumdependent effects of statins," Arteriosclerosis, Thrombosis, and Vascular Biology, vol. 23, no. 5, pp. 729-736, 2003.

[41] U. Laufs, V. La Fata, J. Plutzky, and J. K. Liao, "Upregulation of endothelial nitric oxide synthase by HMG CoA reductase inhibitors," Circulation, vol. 97, no. 12, pp. 1129-1135, 1998.

[42] N. Ghaffari, C. Ball, J. A. Kennedy, I. Stafford, and J. F. Beltrame, "Acute modulation of vasoconstrictor responses by Pravastatin in small vessels," Circulation Journal, vol. 75, no. 6, pp. 1506-1514, 2011.

[43] N. Bao, S. Minatoguchi, H. Kobayashi et al., "Pravastatin reduces myocardial infarct size via increasing protein kinase C-dependent nitric oxide, decreasing oxyradicals and opening the mitochondrial adenosine triphosphate-sensitive potassium channels in rabbits," Circulation Journal, vol. 71, no. 10, pp. 1622-1628, 2007.

[44] S. R. Inman, N. A. Davis, K. M. Olson, and V. A. Lukaszek, "Simvastatin attenuates renal ischemia/reperfusion injury in rats administered cyclosporine A," American Journal of the Medical Sciences, vol. 326, no. 3, pp. 117-121, 2003. 


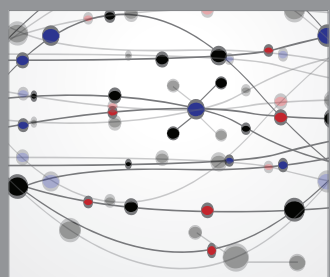

The Scientific World Journal
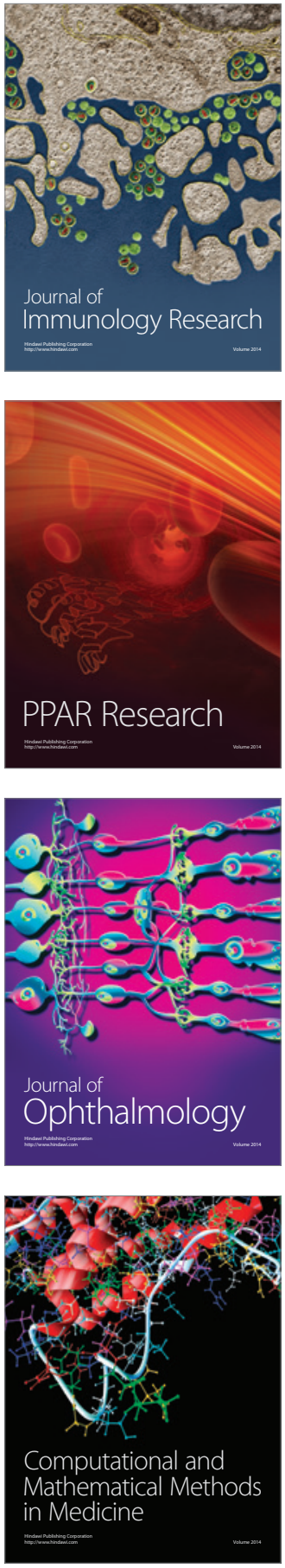

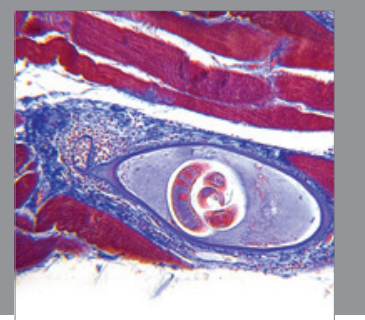

Gastroenterology

Research and Practice
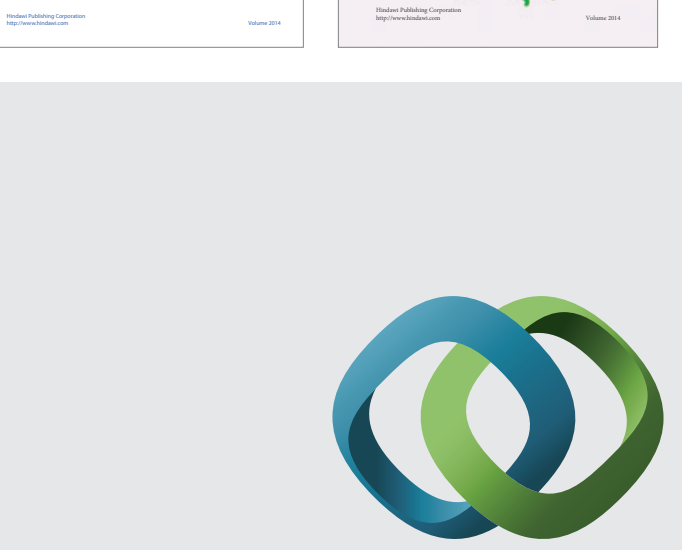

\section{Hindawi}

Submit your manuscripts at

http://www.hindawi.com
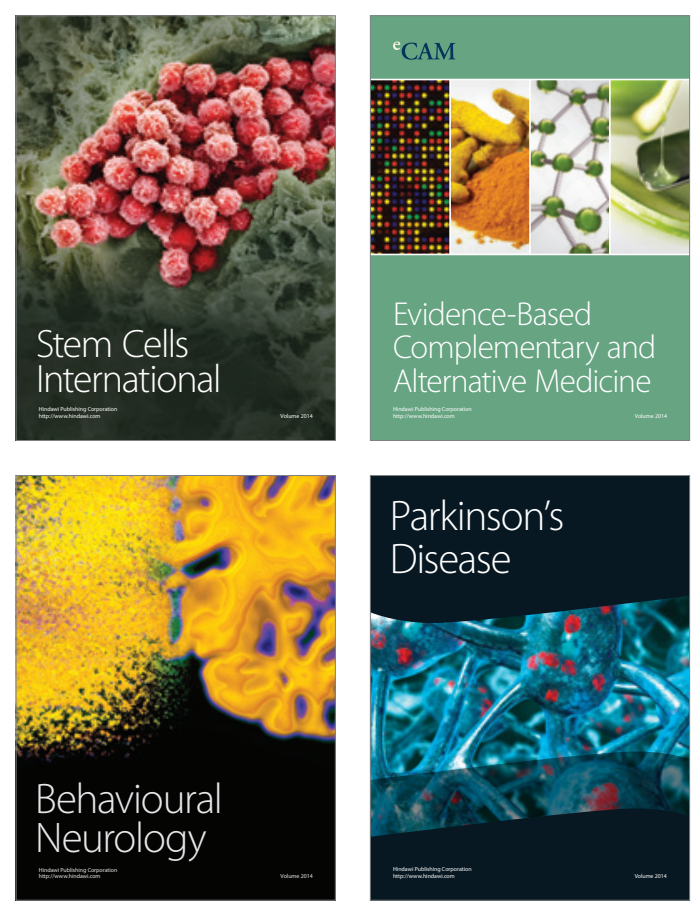

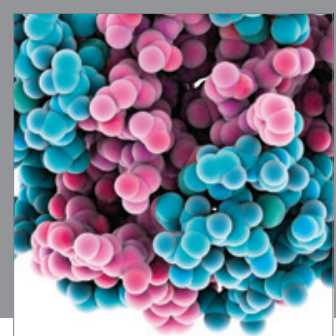

Journal of
Diabetes Research

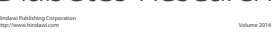

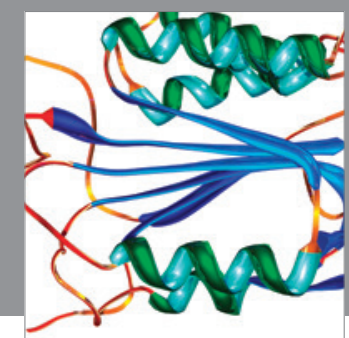

Disease Markers
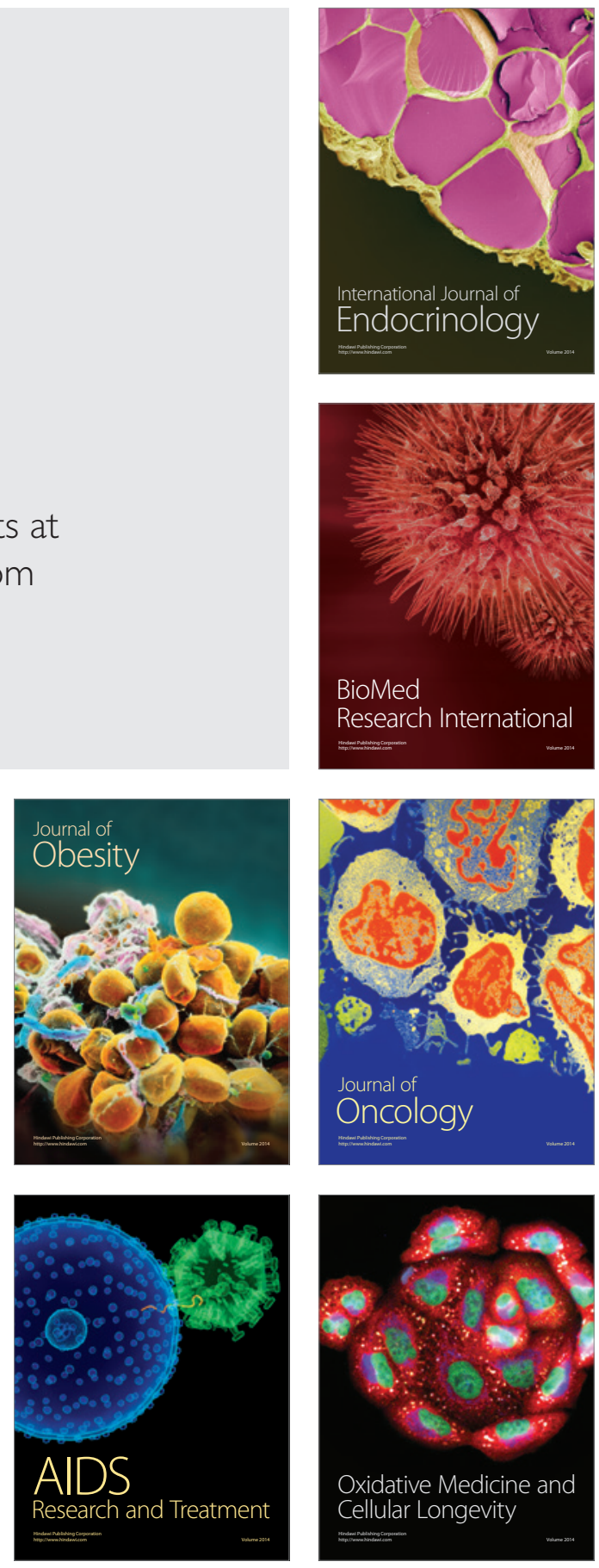